\title{
Motivos para la Práctica del Ejercicio Físico en Estudiantes de Secundaria Reasons for the Practice of Physical Exercise in Mexican Students from Secondary Education \\ *Bertha Alicia Colunga-Rodríguez, **Mario Ángel-González, **Claudia Liliana Vázquez-Juárez, **Julio César Vázquez- Colunga, ${ }^{* *}$ Ramón Castellanos-Ángeles, ${ }^{* *},{ }^{* * * *}$ Cecilia Colunga-Rodriguez, ${ }^{* * *}$ Gabriel Dávalos Picazo \\ *Escuela Normal Superior de Jalisco (México), **Universidad de Guadalajara (México), ***Universidad CEU San Pablo (España), ****Instituto Mexicano del Seguro Social (México)
}

Resumen. Para analizar los motivos para la práctica del ejercicio físico en estudiantes mexicanos de secundaria, se realizó estudio transversal analítico, en 476 participantes. Se aplicó Autoinforme de Motivos para la Práctica del Ejercicio Físico AMPEF (á=.92). para el análisis, se aplicó estadística descriptiva y $\mathrm{Chi}^{2}$. Como resultados, se obtuvo que los promedios de puntajes más altos, fueron para la dimensión de Competición/reconocimiento Social/Desafío $(M=86.54, D E=20.87)$, seguida de Prevención y Salud Positiva $(M=68.21$, $D E=10.74)$ y de Peso e Imagen Corporal $(M=54.40, D E=13.03)$; el puntaje más bajo fue para Control del Estrés $(M=22.39, D E=$ 6.58). Existe significancia estadística entre la variable sociodemográfica de turno, con la dimensión de Urgencias de Salud $($ Chi2 $=32.76$; $p=.008)$, Sexo con Peso e Imagen Corporal (Chi2 $=40.59 ; p=.045)$ y sexo con Fuerza y Resistencia Muscular (Chi2 $=37.73 ; p=.002)$. Destaca el hecho de que la variable de edad, se asoció significativamente con todas las dimensiones del AMPEF y con el total del instrumento $(\mathrm{p}<0.05)$, encontrándose una asociación más fuerte en Competición/Reconocimiento Social/Desafío $($ Chi2 $=179.40, p=$ 0.000), seguida de Prevención y Salud Positiva (Chi2 $=119.74, p=0.000)$ y de Fuerza y Resistencia Muscular $($ Chi2 $=100.98, p=0.000)$, la dimensión que mostró asociación más débil fue Agilidad y Flexibilidad (Chi2 $=42.40, p=0.003)$. Se concluye que es necesario trabajar en los motivos hacia el ejercicio físico en esta población, orientándolos hacia la salud y prevención, para desarrollar estilos de vida sanos y con ellos un mejor desarrollo de los adolescentes.

Palabras clave: motivos, ejercicio físico, adolescentes, escuela secundaria.

Abstract. An analytical cross-sectional study was conducted with 476 Mexican secondary school students to analyze their reasons for the practice of physical exercise. The Self-Report of Reasons for the Practice of Physical Exercise AMPEF (á = .92) was applied. For the analysis, descriptive statistics and Chi2 were applied. As a result, the highest average scores were registered in the Competition / Social recognition / Challenge dimension $(M=86.54, S D=20.87)$, followed by Prevention and Positive Health $(M=68.21, S D=10.74)$ and Weight and Body Image $(M=54.40, S D=13.03)$; the lowest score was found in Stress Control $(M=22.39, S D=6.58)$. There was statistical significance between the sociodemographic variable of shift and the dimension of Health Emergency $($ Chi2 $=32.76 ; p=.008)$, Sex with Weight and Body Image (Chi2 $=40.59 ; p=.045)$ and Sex and Muscular Strength and Resistance $($ Chi2 $=37.73 ; p=.002)$. The age variable was significantly associated with all the dimensions of the AMPEF and with the total of the instrument $(\mathrm{p}<0.05)$, a stronger association having been found with Competition / Social Recognition / Challenge (Chi2 $=179.40, p=0.000)$, followed by Prevention and Positive Health $($ Chi2 $=119.74, p=0.000)$, and Muscular Strength and Resistance $($ Chi2 $=100.98, p=0.000)$. The dimension with the weakest association with age was Agility and Flexibility (Chi2 $=42.40, p=0.003)$. In conclusion, it is necessary to work on the reasons for physical exercise in this population, orienting them towards health and prevention, so to develop healthy lifestyles and by means of those obtaining a better development of the adolescents.

Keywords: reasons, physical exercise, adolescents, high school.

\section{Introducción}

La creciente prevalencia de enfermedades crónicodegenerativas, asociadas a hábitos y estilos de vida (Barba, 2018), ha llamado la atención hacia factores de origen de la enfermedad distintos a los de tipo hereditario, dando más peso a los comportamientos cotidianos de las personas. Entre dichos factores se encuentra el sedentarismo, que aunado a una inadecuada alimentación (Serrano, 2018), contribuye de manera importante a las alarmantes cifras que posicionan a México como uno de los países con mayor prevalencia de obesidad infantil a nivel mundial (Organisation for Economic Cooperation and Development, 2017).

La obesidad infantil es considerada como un factor de riesgo potencial para el desarrollo de enfermedades no transmisibles (ENT), tales como enfermedades cardiovasculares (hipertensión arterial, infarto agudo al miocardio, accidentes cerebrovasculares), cáncer, enfermedades respiratorias (asma y enfermedad pulmonar obstructiva crónica) y diabetes; así

Fecha recepción: 15-03-19. Fecha de aceptación: 30-11-19 Cecilia Colunga-Rodriguez

cecilia.colunga@imss.gob.mx mismo, se ha asociado a la obesidad infantil con muerte prematura y discapacidad en la edad adulta (Organización de las Naciones Unidas para la Alimentación y Agricultura, 2018; Organización Mundial de la Salud (OMS), 2018)

De acuerdo con la OMS, en 2016 había más de 340 millones de niños y adolescentes (de cinco a 19 años) con sobrepeso u obesidad en el mundo, señalando entre sus causas más importantes, a la inadecuada alimentación y al incremento en el sedentarismo (OMS, 2018).

La deficiente práctica de ejercicio físico convierte al sedentarismo en un problema de salud pública (Arocha, 2019). De acuerdo con la Encuesta Nacional de Salud y Nutrición (ENSANUT) de Medio Camino 2016 (Secretaría de Salud, 2016), en México el 39.5\% de adolescentes (de 15 a 19 años) son inactivos, siendo mayor esta proporción en mujeres que en hombres (48.8\% vs 30.1\%). Estos datos sugieren que aproximadamente cuatro de cada 10 adolescentes, no realizan ejercicio físico suficiente para sus requerimientos de salud.

Entre las principales barreras percibidas por adolescentes con sobre peso y obesidad para no realizar ejercicio físico se encuentran la imagen corporal y la ansiedad física so- 
cial (Martínez-Baena, Mayorga-Vega \& Viciana, 2018), la falta de ambientes seguros, el alto costo de los sitios especializados para practicar ejercicio físico, la gran cantidad de tareas y compromisos escolares que tienen los adolescentes, la sensación de perder el tiempo al practicar ejercicio físico, así como la falta de motivación y pereza al realizar esta actividad (Peykari, Eftekhari, Tehrani, Afzali, Hejazi, Atoofi \& Djalalinia, 2015).

A nivel gubernamental, en nuestro país se ha respondido a la necesidad de promover el ejercicio físico con el objetivo de establecer estilos de vida saludables y mejorar el estado de salud en estudiantes de secundaria. De acuerdo con el artículo 30 de la Ley General de Educación (Diario Oficial de la Federación, 30 de septiembre del 2019), los programas y planes de estudio ofertados en las instituciones de educación básica obligatoria (públicos o privados), deberán de incluir el fomento a la actividad física, la práctica del deporte y la educación física, lo anterior como parte de una orientación integral en los educandos. Sin embargo, este espacio curricular se atiende como una responsabilidad escolar, sin incentivar la formación en los menores para que la práctica del ejercicio físico se convierta en parte importante de su estilo de vida.

Ante la necesidad de que los adolescentes adquieran un estilo de vida activo (Jiménez, Moreno, Leyton \& Claver, 2015), se ha investigado la motivación y etapas de cambio para este hábito saludable, buscando en las escuelas públicas de educación básica propiciar las condiciones para que los menores adquieran hábitos saludables. De acuerdo con Fradejas y Espada (2018), la motivación en la edad escolar es imprescindible para lograr que la práctica del deporte sea incorporada como una actividad indispensable en la vida diaria del estudiante; adquieran y mantengan los beneficios físicos y psicológicos que la práctica misma conlleva. Sin embargo, la motivación sigue siendo un punto de partida para incluir en los programas de educación física, y de la cual contamos con elementos teóricos que ayudan a realizar de manera más científico el estudio de este tema.

Según Dosil y Caracuel (como se citó en García, Caracuel \& Ceballos, 2014) la motivación es:

«Un factor disposicional que depende de ciertas características del sujeto, como su condición (física y psíquica) actual o su biografía (gustos, preferencia etc.), así como de objetos o eventos a los que tiende a acercarse o alejarse, de los factores nivel de privación, necesidades, temperatura ambiente, etc., que aumentan o disminuyen en cada momento el valor motivacional, así como de las relaciones actualeshistóricas de ese individuo con sus motivos particulares» (p.72).

En cuanto a los aspectos teóricos que sustentan que la motivación es un elemento fundamental en la realización de actividades humanas, se encuentra la teoría de la autodeterminación formulada por Deci y Ryan (1985), quienes refieren que la conducta intencional del ser humano puede describirse a través de procesos de motivación intrínseca e internalización y que la motivación es uno de los elementos clave para conseguir una adecuada adherencia al ejercicio (Molinero, Salguero \& Márquez, 2011).

Respecto a los motivos de los adolescentes para la práctica de ejercicio, los hallazgos de Jiménez et al. (2015) sugie- ren que la necesidad psicológica básica de competir es la variable más relevante para adherirse a los estadios de práctica de ejercicio físico más activos. De acuerdo con González y Portolés (2014) existen diferencias estadísticamente significativas entre hombres y mujeres que realizan actividad física y deportiva extraescolar por lo menos un día a la semana, siendo los hombres con un $50.35 \%$ y las mujeres con $30.16 \%$, asimismo mencionan que la actividad física va disminuyendo en los adolescentes en la medida que van avanzando en los cursos de primero al último grado de la Educación Secundaria Obligatoria. Por otro lado, los adolescentes que practican por lo menos un día actividad física extracurricular puntúan más bajo en el consumo de alcohol, tabaco y cannabis y obtienen mejores calificaciones en matemáticas, lengua castellana y educación física.

Jiménez, et al. (2015) realizaron un estudio con el objetivo de encontrar algunos factores predictores para el ejercicio físico en adolescentes; para ello aplicaron el Cuestionario de Estadios de Cambio para el Ejercicio Físico - Medida Continua (URICA-E2), es un instrumento de 24 ítems, que evalúa los estadios de cambio del ejercicio físico; dicho cuestionario fue diseñado por Marcus, Selby, Niaura y Rossi (1992) y validado y traducido al español por Moreno en el 2010. Para fines de investigación, Jiménez et al. (2015) extrajeron tres factores del URICA-E2: precontemplación (consideraciones para poder realizar ejercicio físico, aunque éste no se realizará), contemplación-preparación (reflexionar el practicar ejercicio físico de manera regular), y acción-mantenimiento (reconocer que se ha logrado realizar ejercicio físico durante un tiempo determinado). Los autores encontraron que el estadio de precontemplación fue predicho negativamente por la competencia y positivamente por la desmotivación y percepción del clima motivacional que implica el ego; el estadio de contemplación-preparación fue predicho positivamente por la competencia y la motivación intrínseca-perfección, la motivación extrínseca-introyectada, y negativamente por la autonomía, y el estadio de acción-mantenimiento fue predicho positivamente por la competencia. Estos resultados sugieren que la necesidad psicológica básica de competencia es la variable más relevante para adherirse a los estadios de práctica de ejercicio físico más activos.

Portela y Domínguez (2017) realizaron un estudio en el cual aplicaron el cuestionario de Autoinforme de Motivos para la Práctica de Ejercicio Físico en una población de 342 estudiantes de Educación Secundaria Obligatoria y Bachillerato, en donde encontraron que los factores prevención/ salud positiva y diversión/bienestar, son los principales factores motivacionales en la práctica del ejercicio físico. Mientras que los factores con menor impacto en la motivación son en el reconocimiento social/desafío y urgencias de salud. Resultados semejantes obtuvieron Tárrega-Canós, Alguacil y Parra-Camacho (2018), quienes afirman que tanto en hombres como en mujeres el factor con mayor valoración es el de prevención/salud positiva, bienestar/diversión y fuer$\mathrm{za} /$ resistencia muscular, sin embargo, el factor al que menos importancia le otorgan es, para las mujeres, el de reconocimiento social y para los hombres el de emergencias de salud. Estos autores mencionan que en la medida que los estudiantes avanzan de ciclo escolar disminuye la actividad física extraescolar, es decir, que entre mayor es el grado escolar es 
menor el ejercicio físico.

Portela (2018) encontraron que las principales variables predictoras motivacionales son competencia, reconocimiento social/desafío (16.1\%), fuerza/resistencia muscular (13.3\%) y prevención/salud positiva (12\%). Las barreras para la práctica del ejercicio físico con una gran influencia predictora son fatiga/pereza (14.9\%), imagen corporal/ansiedad físicosocial $(15 \%)$ y obligaciones/falta de tiempo (3.6\%). Tárrega, et al. (2018) no encontraron diferencia en la motivación entre quienes, si hacen ejercicio y no hacen ejercicio, fuera del contexto escolar.

Los resultados hasta aquí descritos plantean contradicciones y poca claridad respecto a los motivos que llevan a los adolescentes a realizar ejercicio físico, particularmente en las escuelas secundarias públicas, donde se ubica el mayor porcentaje de la población de esta edad. Adicionalmente destaca la importancia que tiene la motivación para la práctica del ejercicio físico para el sano desarrollo de los adolescentes y el rol que juega la escuela secundaria, en la formación de hábitos saludables, reconociendo el valor disposicional de los motivos en estas prácticas del estilo de vida. Dado lo anterior, este estudio tiene como principal propósito, identificar los principales motivos que tienen los adolescentes para la práctica de ejercicio físico, lo cual puede ser el punto de partida para planear acciones que incrementen su práctica y con ello, la promoción de hábitos y estilos de vida saludables (Vera-Estrada, Sánchez-Rivas \& SánchezRodríguez, 2018), para el mejoramiento en la condición física (Galmes-Panades \& Vidal-Conti, 2020), la disminución de la tasa de mortalidad temprana y el riesgo cardiovascular (Blanco, Soto, Benitez, Modaca \& Jurado, 2019), entre otras.

\section{Método}

Diseño de estudio: transversal y analítico.

\section{Participantes}

Se incluyó por medio de censo, 476 adolescentes de una secundaria pública en la zona metropolitana de Guadalajara, en México. Se integraron a la muestra por medio de casos consecutivos (Otzen, T. \& Manterola C., 2017). El periodo en que se realizó el estudio fue en el primer semestre del año 2018.

\section{Instrumentos}

Se aplicó una cédula de datos generales elaborada específicamente para este estudio, donde los estudiantes registraron datos como edad, género, grado escolar y horario de asistencia a la escuela (turno), así como el instrumento Autoinforme de Motivos para la Práctica del Ejercicio Físico (AMPEF), validado por Domínguez-Alonso, LópezCastelo y Portela-Pino (2018), escala adaptada al español por Capdevila, Niñerola y Pintanel (2004) a partir del Ejercise Motivations Inventory. El cuestionario presenta el siguiente encabezado: «personalmente practico (o practicaría) ejercicio físico»; consta de cuarenta y ocho reactivos agrupados en once factores: peso e imagen corporal (siete ítems), diversión y bienestar (seis ítems), prevención y salud positiva (seis ítems), competición (cuatro ítems), afiliación (cuatro ítems), fuerza y resistencia muscular (cuatro ítems), recono- cimiento social (cuatro ítems), control del estrés (tres ítems), agilidad y flexibilidad (tres ítems), desafío (cuatro ítems), y urgencias de salud (tres ítems). Su formato de respuesta es tipo Likert de cero (nada verdadero para mí) a diez (totalmente verdadero para mí). El instrumento fue estandarizado en población de adolescentes gallegos de educación secundaria y mostró una varianza total explicada del $65.24 \%$ y un ajuste adecuado a los datos $\left(X^{2} / \mathrm{df}=2.635, \mathrm{GFI}=.909, \mathrm{CFI}=\right.$ .901$, RMSEA $=.047)$, así como una confiabilidad para todo el instrumento por alfa de Cronbach de .96, las once dimensiones iniciales mencionadas por Capdevila et al. (2004) se agruparon en ocho, que son Prevención y Salud Positiva (ocho ítems), Competición/Reconocimiento Social/Desafío (doce ítems), Peso e Imagen Corporal (siete ítems), Afiliación, Diversión y Bienestar (siete ítems), Control del estrés (tres ítems), Fuerza y Resistencia Muscular (cuatro ítems), Urgencias de Salud (cuatro ítems) y Agilidad y Flexibilidad (tres ítems), (Domínguez, López \& Portela, 2018). La forma en que se calificaron las dimensiones y el global del instrumento, fue sumando los puntajes asignados.

\section{Procedimiento}

La aplicación de los instrumentos se realizó de manera colectiva, fueron contestados por los participantes de manera individual, durante el horario de clases. Se solicitó autorización a los directivos del plantel y a los padres de familia, exponiendo los objetivos del estudio y señalando la confidencialidad de los datos y la participación voluntaria en el estudio, sin ninguna consecuencia. Se tomó asentimiento a los adolescentes.

\section{Análisis de los datos}

Se utilizó el programa estadístico Statistical Package for the Social Sciences (SPSS), versión 21.0. Se realizó análisis descriptivo usando frecuencias simples, media, desviación estándar y porcentajes. Para determinar la asociación entre variables nominales y dimensiones, se aplicó la prueba Chi cuadrado, tomando como significativo, un valor de $p<$ .05 .

\begin{tabular}{lccc}
$\begin{array}{l}\text { Tabla } 1 . \\
\text { Características de la muestra }\end{array}$ & & \\
\hline Variable & Femenino & Frecuencia & Porcentaje \\
\multirow{2}{*}{ Sexo } & Masculino & 243 & 52.7 \\
& $1^{\circ}$ & 225 & 47.3 \\
Grado & $2^{\circ}$ & 153 & 32.1 \\
& $3^{\circ}$ & 162 & 34.0 \\
Turno & Matutino & 250 & 33.6 \\
& Vespertino & 225 & 52.7 \\
\hline Fuente. Cédula de datos $\mathrm{N}=476$ & & & \\
\end{tabular}

\section{Resultados}

De los 476 estudiantes del censo de la escuela, el sexo fue en su mayoría femenino, la edad promedio fue de 12.30 $(D E=1.02$ años). La mayoría pertenecían al segundo grado (34\%) y más de la mitad correspondió al turno matutino $(52.7 \%)$ (Tabla 1$)$.

Como se puede apreciar en la tabla 2, los promedios más altos fueron para la dimensión de Competición/reconocimiento Social/Desafío $(M=86.54, D E=20.87)$, seguida de Prevención y Salud Positiva $(M=68.21, D E=10.74)$ y de Peso e Imagen Corporal $(M=54.40, D E=13.03)$; el puntaje más bajo 
Tabla 2.

\begin{tabular}{|c|c|c|c|c|}
\hline Dimensiones & Mínimo & Máximo & $\mathrm{M}$ & $\mathrm{DE}$ \\
\hline Prevención y Salud Positiva & 16 & 80 & 68.21 & 10.764 \\
\hline Competición/Reconocimiento Social/Desafío & 24 & 120 & 86.54 & 20.879 \\
\hline Peso e Imagen Corporal & 14 & 70 & 54.40 & 13.039 \\
\hline Afiliación, Diversión y Bienestar & 14 & 70 & 51.51 & 12.191 \\
\hline Control del estrés & 6 & 30 & 22.39 & 6.581 \\
\hline Fuerza y Resistencia Muscular & 8 & 40 & 33.23 & 6.778 \\
\hline Urgencias de Salud & 8 & 40 & 26.42 & 8.543 \\
\hline Agilidad y Flexibilidad & 6 & 30 & 24.39 & 5.769 \\
\hline Total & 114 & 480 & 367.10 & 63.493 \\
\hline \multicolumn{5}{|l|}{ Fuente: AMPEF N=476 } \\
\hline \multicolumn{5}{|c|}{$\begin{array}{l}\text { Tabla } 3 \text {. } \\
\text { Asociación entre dimensiones del AMPEF y variables sociodemográficas }\end{array}$} \\
\hline Dimensión & Grado escolar & Turno & Sexo & Edad \\
\hline Prevención y Salud Positiva & 51.105 & 14.35 & 22.20 & $119.74 * *$ \\
\hline Competición/Reconocimiento Social/Desafío & 87.54 & 47.10 & 49.68 & $179.40^{* *}$ \\
\hline Peso e Imagen Corporal & 41.62 & 26.43 & $40.59 *$ & $76.12 *$ \\
\hline Afiliación, Diversión y Bienestar & 54.88 & 26.87 & 39.15 & $98.47 * *$ \\
\hline Control del estrés & 34.90 & 5.71 & 7.71 & $53.60 * *$ \\
\hline Fuerza y Resistencia Muscular & 26.15 & 14.99 & $37.73^{* *}$ & $100.98^{* *}$ \\
\hline Urgencias de Salud & 26.72 & $32.76^{* *}$ & 17.97 & $54.03 * *$ \\
\hline Agilidad y Flexibilidad & 20.64 & 5.67 & 12.36 & $47.40^{* *}$ \\
\hline Total & 247.80 & 115.32 & 118.79 & $479.44 * *$ \\
\hline
\end{tabular}

fue para Control del Estrés $(M=22.39, D E=6.58)$ (Tabla 2).

Por otra parte, la tabla 3 , muestra asociación de significancia estadística entre la variable sociodemográfica de turno, con la dimensión de Urgencias de Salud $\left(C h i^{2}=\right.$ 32.76; $p=.008)$, Sexo con Peso e Imagen Corporal $\left(C h i^{2}=\right.$ $40.59 ; p=.045)$ y sexo con Fuerza y Resistencia Muscular $\left(C h i^{2}=37.73 ; p=.002\right)$. Destaca el hecho de que la variable de edad, se asoció significativamente con todas las dimensiones del AMPEF y con el total del instrumento $(p<.05)$, encontrándose una asociación más fuerte en Competición/ Reconocimiento Social/Desafío $\left(C h i^{2}=179.40, p=.000\right)$, seguida de Prevención y Salud Positiva $\left(C h i^{2}=119.74, p=\right.$ $.000)$ y de Fuerza y Resistencia Muscular $\left(C h i^{2}=100.98, p=\right.$ .000 ), la dimensión que mostró asociación más débil fue Agilidad y Flexibilidad $\left(C h i^{2}=42.40, p=.003\right)$ (Tabla 3$)$.

\section{Discusión}

El presente estudio, tuvo como principal objetivo analizar los motivos para la práctica del ejercicio físico en estudiantes de secundaria. Respecto a las características sociodemográficas, encontramos que al igual que en otros estudios, la mayoría son mujeres, de 12 años, que acuden a la escuela secundaria en el turno matutino (Aspano, Lobato, Leyton, Batista \& Jiménez, 2016; Fradejas \& Espada, 2018; Franco, Coterón \& Gómez, 2017; Domínguez, López \& Portela, 2018).

Respecto a los motivos para la práctica del ejercicio físico, encontramos promedios más altos en las dimensiones de Competición/Reconocimiento Social/Desafío, seguida de Prevención y Salud Positiva y de Peso e Imagen Corporal; estos resultados contrastan con los obtenidos por Portela y Domínguez (2017), quiénes encontraron que los principales factores motivacionales para la práctica del ejercicio físico en adolescentes gallegos fueron la Prevención/Salud Positiva y Diversión/Bienestar. En cuanto al factor con el puntaje más bajo en nuestro estudio fue Control del Estrés, en tanto que en el realizado por Portela y Domínguez (2017) fueron el Reconocimiento Social/Desafío y Urgencias de Salud; esto pudiera sugerir que el contexto cultural juega un papel muy importante en la motivación hacia el ejercicio físico.

En cuanto a las asociaciones estadísticas entre las variables sociodemográficas y las dimensiones del AMPEF, en- contramos asociación de significancia estadística entre la variable sociodemográfica de turno, con la dimensión de Urgencias de Salud, Sexo con Peso e Imagen Corporal y sexo con Fuerza y Resistencia Muscular, esto concuerda con los datos obtenidos por López y López (2015), quienes encontraron diferencias con significancia estadística con el Sexo en las dimensiones de Peso e Imagen Corporal, Fuerza y Resistencia muscular.

La variable de edad, destacó en este estudio, debido a que se asoció significativamente con todas las dimensiones del AMPEF y con el total del instrumento $(p<.05)$, encontrándose una asociación más fuerte en Competición/Reconocimiento Social/Desafío, seguida de Prevención y Salud Positiva) y de Fuerza y Resistencia Muscular, la dimensión que mostró asociación más débil fueAgilidad y Flexibilidad lo cual indica que la edad juega un papel importante en la práctica del ejercicio físico, puesto que modula el interés y los motivos que incitan al adolescente a realizar este tipo de actividad en su vida diaria (Martínez, et al., 2018).

Entre las fortalezas del estudio se encuentra el que se realizó por censo, aunque no representa estadísticamente a la población de adolescentes del país, sin embargo, al tratarse de una escuela pública, en una zona económica media, permite tener una referencia de los motivos que refieren adolescentes de escuelas similares en nuestro país.

El estudio de la motivación para la práctica deportiva en adolescentes mexicanos tiene escasos referentes, de ahí la importancia del presente estudio, no así es el caso de múltiples estudios reportados en España, lo cual podría ser indicativo de una distinta cultura del ejercicio físico que en general provea a los adolescentes de una forma distinta de conceptualizar la práctica cotidiana del ejercicio y por tanto, se refleje en distintas motivaciones.

Los resultados del presente estudio sugieren diferencias por género y por turno en cuanto a factores motivacionales, no obstante, otros estudios no reportan estas diferencias, que al menos en población mexicana, se requiere profundizar, con distintos diseños y con muestras más grandes que representen a la población mexicana. Respecto a diferencias por género, destacan los mayores puntajes en hombres, que pueden deberse a los roles sociales de masculinidad que en la población mexicana prevalecen relacionados con la práctica de actividades deportivas más en hombres que en mujeres y que en las escuelas secundarias pudiera estar prevaleciendo, por lo que amerita profundizar con enfoque de género y con estudios cualitativos, el significado de la práctica del ejercicio físico en mujeres mexicanas, ya que nuestra población muestra un problema grave de obesidad en mujeres, que pudiera estar relacionado con roles sociales femeninos de mayor sedentarismo.

En el factor Competición, Tarrega, et al. (2018), encontraron diferencias en función del género, en este estudio, se encontró asociación entre la dimensión Competición y diferencia estadística en la variable Turno, lo cual puede ser indicativo de aspectos sociales del entorno, que se manifiestan distintos por los horarios escolares, en otras palabras, tal parece que los adolescentes del turno vespertino, indistintamente al sexo, perciben como una motivación importante la competición y la agilidad y flexibilidad probablemente porque se requiere para las amenazas del contexto en la tarde, ya 
que la salida de la escuela secundaria, se da en el inicio de la noche y la mayoría de los adolescentes de las escuelas públicas, se trasladan a sus casas caminando.

En cuanto a aspectos teóricos que proporcionen bases científicas, tanto para la realización de investigaciones en el tema, como para la implementación de programas de intervención que incentiven la motivación para la práctica de ejercicio físico, la teoría de la autodeterminación, propuesta por Deci y Ryan (1985), resulta aplicable a población adolescente, ya que por la etapa de desarrollo madurativo que presenta este grupo poblacional, tienen la capacidad de intencionar su conducta por medio de procesos intrínsecos e internalizantes, donde la motivación resulta un elemento esencial para favorecer la adherencia a estilos de vida saludables.

En términos de aplicaciones prácticas, los hallazgos de este estudio, pueden favorecer el diseño de programas centrados en aspectos psicológicos de la práctica en el ejercicio físico y el deporte, que desde las escuelas secundarias, incluyan en las clases de educación física, factores motivacionales tendientes a favorecer la condición física y la salud integral, que tengan un alto contenido lúdico orientado al disfrute y bienestar, a nivel individual y colectivo, en un ambiente escolar favorable para la cultura del deporte

\section{Conclusiones}

Los resultados muestran predominio en puntajes más altos en la dimensión de Competición/reconocimiento Social/Desafío, seguida de Prevención y Salud Positiva y de Peso e Imagen Corporal; el puntaje más bajo fue para Control del Estrés. Asimismo, se encontró asociación de significancia estadística entre la variable sociodemográfica de turno, con la dimensión de Urgencias de Salud $(p<.05)$, Sexo con Peso e Imagen Corporal $(p<.05)$ y sexo con Fuerza y Resistencia Muscular $(p<.05)$. La edad, se asoció con todas las dimensiones del AMPEF y con el total del instrumento $(p<.05)$, encontrándose una asociación más fuerte en Competición/ Reconocimiento, seguida de Prevención y Salud Positiva y de Fuerza y Resistencia Muscular, la dimensión que mostró asociación más débil fue Agilidad y Flexibilidad. Los hallazgos sugieren la necesidad de trabajar en el desarrollo de motivos para la práctica del ejercicio físico en los estudiantes de secundaria, centrando el interés en la salud y prevención.

\section{Conflictos de interés}

Los autores declaran que no existe ningún conflicto de interés.

\section{Referencias}

Arocha, I. (2019). Sedentarismo, la enfermedad del siglo XXI. Clínica e Investigación En Arteriosclerosis, 31(5), 233 240. doi: 10.1016/j.arteri.2019.04.004

Aspano, M., Lobato, S., Leyton, M., Batista, M., \& Jiménez, R. (2016). Predicción de la motivación en las etapas de cambio de ejercicio más activos. Retos, (30), 87-91. Recuperado de http://www.redalyc.org/ articulo.oa?id $=345744747016$

Barba, J. (2018). México y el reto de las enfermedades crónicas no transmisibles. El laboratorio también juega un papel importante. Revista Latinoamericana de Patología Clínica y Medicina de Laboratorio, 65(1), 4-17. Recuperado de https://www.medigraphic.com/cgibin/new/ resumen.cgi?IDARTICULO=79689

Blanco, J., Soto, M., Benitez, Z., Mondaca, F. \& Jurado, P. (2019). Barreras para la práctica de ejercicio físico en universitarios mexicanos comparaciones por género. Retos, (36), 80-82. Recuperado de https://recyt.fecyt.es/ index.php/retos/article/view/67820

Capdevila, Ll., Niñerola, J., \& Pintanel, M. (2004). Motivación y actividad física: el autoinforme de motivos para la práctica de ejercicio físico (AMPEF). Revista de Psicología del Deporte, 13(1), 55-74. Recuperado de https:// core.ac.uk/download/pdf/13296214.pdf

Deci, E. \& Ryan, R. (1985). Intrinsic Motivation and SelfDetermination in Human Behavior (1st ed.). Springer US. doi: 10.1007/978-1-4899-2271-7

Diario Oficial de la Federación. (30 de septiembre del 2019). Ley General de Educación. Recuperado de

http://www.diputados.gob.mx/LeyesBiblio/pdf/ LGE_300919.pdf

Domínguez-Alonso, J., López-Castedo, A. \& Portela-Pino, I. (2018). Predictive variables of motivation and barriers for the practice of physical exercise in adolescence. Journal of Human Sport and Exercise, in press. doi: 10.14198/ jhse.2018.134.17

Fradejas, E. y Espada, M. (2018). Evaluación de la motivación en adolescentes que practican deportes en edad escolar. Retos, (33), 27-33.

Franco, E., Coterón, J. \& Gomez, V. (2017). Promoción de la actividad física en adolescentes: rol de la motivación y autoestima. Psiencia. Revista Latinoamericana De Ciencia Psicologica, 9(2), 15. doi: 10.5872/psiencia/9.2.24

Galmes-Panades, A. \& Vidal-Conti, J. (2020). Cómo fomentar la práctica de ejercicio físico a través de los deberes activos en estudiantes universitarios. Retos, (37), 576-584. Recuperado de https://recyt.fecyt.es/index.php/retos/ article/view/ 72110

García, J., Caracuel, J. \& Ceballos, O. (2014). Motivación Y Ejercicio Físico Deportivo: Una Añeja Relación. Revista Internacional de Ciencias Sociales y Humanidades, SOCIOTAM, XXIV(1), 71-88.

González, J. \& Portolés, A. (2014). Actividad física extraescolar: relaciones con la motivación educativa, rendimiento académico y conductas asociadas a la salud. Revista Iberoamericana de Psicología del Ejercicio y el Deporte, 9 (1), 51-65. Recuperado de https://www.redalyc.org/pdf/ 3111/311130199005.pdf

Jiménez, R., Moreno, B., Leyton, M. \& Claver, F. (2015). Motivación y estadios de cambio para el ejercicio físico en adolescentes. Revista Latinoamericana de Psicología, 47(3), 196-204. doi:10.1016/j.rlp.2014.11.001 0120-0534

López,A. \& López, E. (2015). Variables motivacionales implicadas en la actividad física en el alumnado de secundaria. Revista de Estudios e Investigación En Psicología y Educación, (1). doi: 10.17979/reipe.2015.0.01.169

Marcus, B. H., Selby, V. C., Niaura, R. S. \& Rossi, J. S. (1992). 
Self-efficacy and the stages of exercise behavior change. Research Quarterly for Exercise and Sport, 63(1), 6066. doi: 10.1080/02701367.1992.10607557

Martínez-Baena, A., Mayorga-Vega, D. \& Viciana, J. (2018). Factores predictores de la actividad física en escolares españoles de acuerdo a su estado de peso. Retos, (33), $74-80$

Molinero, O., Salguero, A., \& Márquez, S. (2011). Autodeterminación y adherencia al ejercicio: estado de la cuestión. Revista Internacional de Ciencias del Deporte, 25(7), 287-304. doi:10.5232/ricyde2011.02504

Organisation for Economic Cooperation and Developmen. (2017). Obesity Update 2017. Recuperado de http:// fmdiabetes.org/wp-content/uploads/2017/08/La-OCDEpresentoì-el-informe-de-actualizacioìn-sobre-la-obesidad-2017.pdf

Organización de las Naciones Unidas para la Alimentación y Agricultura. (2018). FAO presenta buenas prácticas para reducir sobrepeso y obesidad en niñas y niños escolares. Recuperado de http://www.fao.org/mexico/noticias/ detailevents/

Organización Mundial de la Salud (2018). Obesidad y www.who.int/es/news-room/fact-sheets/detail/obesityand-overweight

Otzen, T. \& Manterola, C. (2017). Técnicas de Muestreo sobre una Población a Estudio. International Journal of Morphology, 35(1), 227-232. doi: 10.4067/S071795022017000100037.

Peykari, N., Eftekhari, M. B., Tehrani, F. R., Afzali, H. M., sobrepeso. Datos y cifras. Recuperado de https://

Hejazi, F., Atoofi, M. K. \& Djalalinia, S. (2015). Promoting physical activity participation among adolescents: The barriers and the suggestions. International Journal of Preventive Medicine, 6(12). doi. 10.4103/2008-7802.151820

Portela, I. (2018). Análisis de la motivación hacia la actividad física y barreras para su práctica en adolescentes gallegos (Tesis doctoral). Universidad de Vigo, España. Recuperado de http://www.investigo.biblioteca.uvigo.es/ xmlui/ handle/11093/977

Portela, I. \& Domínguez, J. (2017). Motivación percibida por los adolescentes gallegos en la práctica del ejercicio. Revista de Estudios e Investigación En Psicología y Educación, (14), 117-121. doi: 10.17979/ reipe.2017.0.14.2518

Secretaría de Salud. (2016). Encuesta Nacional de Salud y Nutrición de Medio Camino 2016. Recuperado de https://ensanut.insp.mx/encuestas/ensanut2016/doctos/ informes/ENSANUT2016ResultadosNacionales.pdf

Serrano, J. (2018). La obesidad infantil y juvenil. Quaderns de Polítiques Familiars, (4), 36-47. Recuperado de http:/ /repositori.uic.es/handle/20.500.12328/958

Tárrega-Canós, J., Alguacil, M. \& Parra-Camacho, D. (2018). Análisis de la Motivación hacia la Práctica de Actividad Física Extraescolar en Educación Secundaria. Multidisciplinary Journal of Educational Research, 8(3), 259-280. doi:10.17583/remie.2018.3754

Vera-Estrada, F., Sánchez-Rivas, E. \& Sánchez-Rodríguez, J. (2018). Promoción de la actividad física saludable en el recreo escolar. Revista Internacional de Medicina y Ciencias de La Actividad Fisica y Del Deporte, 18(72), 655668. doi: 10.15366/rimcafd2018.72.004

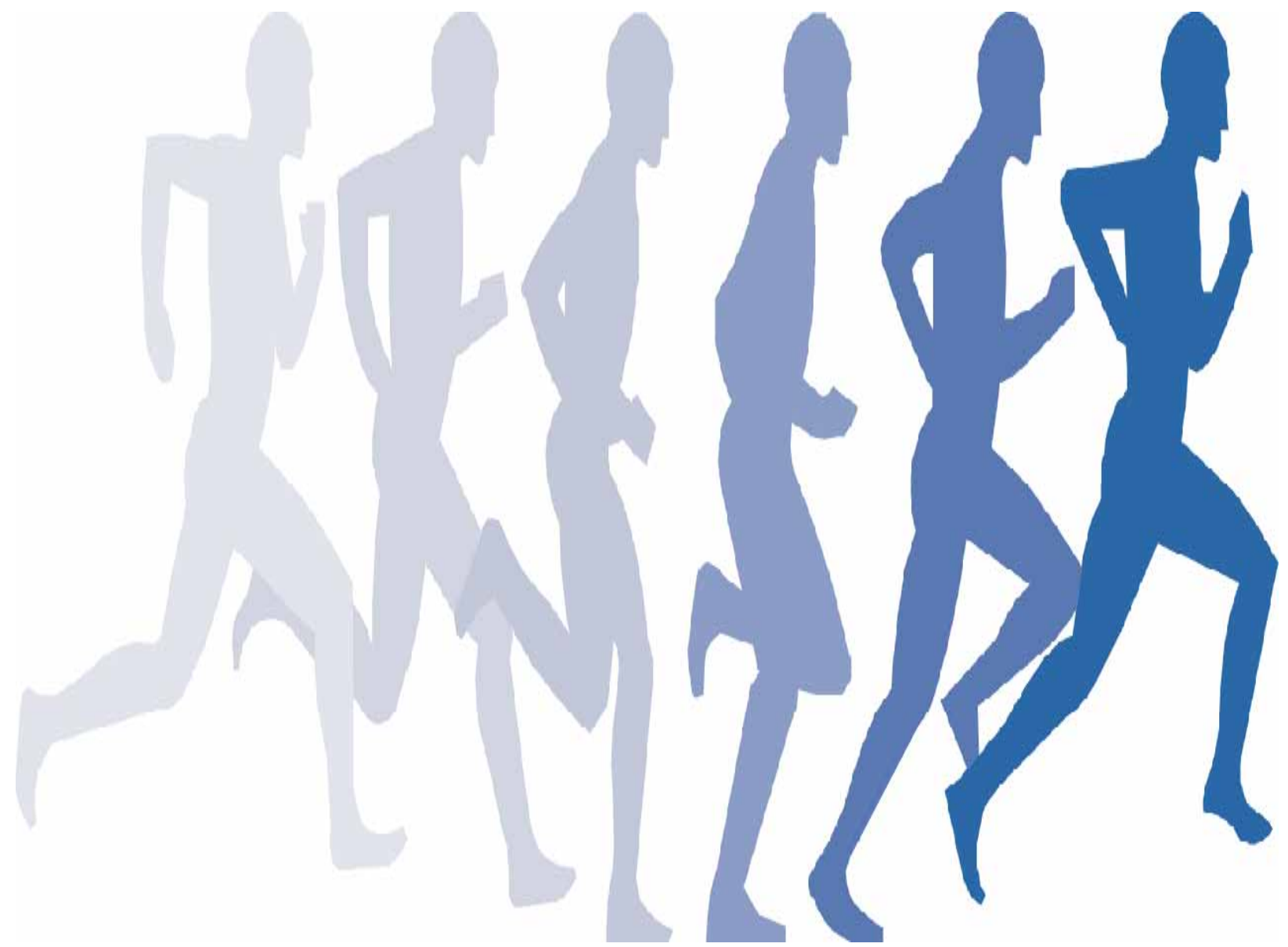

